\title{
La question du dissensus au Parlement européen : le contre-discours de l'opinion minoritaire
}

The issue of dissensus in the European Parliament: the counter-discourse of the minority opinion

\section{Rachele Raus}

\section{(2) OpenEdition}

\section{Journals}

Édition électronique

URL : http://journals.openedition.org/aad/2621

DOI : 10.4000/aad.2621

ISSN : $1565-8961$

Éditeur

Université de Tel-Aviv

Référence électronique

Rachele Raus, « La question du dissensus au Parlement européen : le contre-discours de l'opinion minoritaire », Argumentation et Analyse du Discours [En ligne], 21 | 2018, mis en ligne le 15 octobre 2018, consulté le 23 septembre 2019. URL : http://journals.openedition.org/aad/2621 ; DOI : 10.4000/ aad.2621

Ce document a été généré automatiquement le 23 septembre 2019

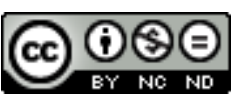

Argumentation \& analyse du discours est mis à disposition selon les termes de la licence Creative Commons Attribution - Pas d'Utilisation Commerciale - Pas de Modification 4.0 International. 


\section{La question du dissensus au Parlement européen : le contre- discours de l'opinion minoritaire}

The issue of dissensus in the European Parliament: the counter-discourse of the minority opinion

Rachele Raus

\section{Introduction}

1 Comment un contre-discours peut-il s'exprimer sur le plan institutionnel dans des démocraties «de consensus » (Bendjaballah 2014) où le «lissage énonciatif » (Oger, Ollivier-Yaniv $2006: 64$ ) caractérise le discours institutionnel - lequel finit souvent par recourir au flou et à l'ambiguïté (Krieg-Planque 2012 : 155) pour pouvoir s'adresser à des publics diversifiés et à des auditoires fort hétérogènes? Il faut bien voir, en effet, que ce lissage n'implique pas forcément l'absence de points de vue divergents.

2 En tant que discours institutionnel (Oger, Yaniv 2003, Krieg-Planque 2012), le discours $\mathrm{du}$ Parlement européen (désormais PE) est un exemple révélateur de la coprésence du lissage énonciatif et de la persistance de positionnements divergents (Raus 2015, 2017). Dans son cadre, en effet, « le consensus ne traduit pas nécessairement l'obtention d'un accord sur l'intégralité des dispositions du texte débattu» (Bendjaballah 2014: 68). Certains désaccords subsistent, qui deviennent normalement visibles dans les «opinions minoritaires». Ces opinions minoritaires peuvent être formulées explicitement à l'intérieur des rapports parlementaires non législatifs, comme prévu par le Règlement du PE (art. 46 de 2004 ; art. 52 de 2009 ; art. 56 de 2016 ; art. 56 bis de 2017).

Dans ce contexte, nous nous proposons d'analyser les opinions minoritaires de la commission des affaires étrangères et de la commission des droits de la femme et de l'égalité des genres de l'actuelle législature. Nous partons pour ce faire d'une 
« conception englobante» de l'argumentation, selon laquelle (Amossy et Koren 2010 : 14) « l'argumentation politique relève aussi bien de la recherche du consensus (but que lui assignent la plupart des théories de l'argumentation) que de la gestion du dissensus qui est au cœur de la vie politique, par définition conflictuel ».

Il s'agit de voir comment les opinions minoritaires peuvent faire circuler des contrediscours polémiques à l'intérieur du PE et alimenter le dissensus indispensable à tout débat démocratique. Pour ce faire, nous présentons d'abord les caractéristiques de l'opinion minoritaire comme discours monogéré pour ensuite distinguer deux types d'avis minoritaire: individuel et collectif. Par rapport à ces deux catégories, nous analyserons deux cas spécifiques : le premier concernant les rapports sur la défense et la sécurité nous permettra de mieux voir les modalités de l'avis collectif; le deuxième, qui a trait aux rapports sur la prostitution et sur l'égalité entre les hommes et les femmes, nous fera mieux observer les avis individuels. Bien que les exemples choisis portent sur des sujets distincts, ils nous permettront de montrer en quoi les avis individuels et collectifs véhiculent la polémique de manière différente tout en partageant certains traits communs.

\section{Préliminaires : l'opinion minoritaire comme discours monogéré}

5 L'expression de l'opinion minoritaire est prévue dans le règlement de la huitième législature du PE dont l'art. 56, dans sa version de 2016, énonce :

Si l'avis de la commission n'est pas unanime, le rapport doit également faire état des opinions minoritaires. Exprimées à l'occasion du vote sur l'ensemble du texte, celles-ci peuvent, sur demande de leurs auteurs, faire l'objet d'une déclaration écrite d'un maximum de 200 mots, annexée à l'exposé des motifs.

Un an plus tard, l'article est légèrement modifié dans l'art. 56bis: "Des opinions minoritaires peuvent être exprimées à l'occasion du vote sur l'ensemble du texte et peuvent, sur demande de leurs auteurs, faire l'objet d'une déclaration écrite de 200 mots au maximum, annexée à l'exposé des motifs. " Les règlements de la huitième législature du PE ne changent pas grand-chose à l'expression de l'opinion minoritaire d'un point de vue formel, mais le renforcement des groupes politiques et du Président du PE a eu comme conséquence la limitation de l'influence des députés à titre individuel aussi bien que celle des opinions minoritaires.

7 Expression du dissensus, l'opinion « minoritaire » est résiduelle dans un système qui, à la différence de l'argumentation talmudique, n'a pas développé « a system of logic and inference that could tolerate ambiguous or multiple identities, contradictions, and allowed for the possibility of bivalent and multivalent values or entities» (Frank 2003 : 180). Cependant, nous verrons que tout en restant dans les marges, l'opinion minoritaire n'en joue pas moins un rôle fondamental dans le débat public.

Précisons que, bien qu'il soit prononcé devant le PE à l'occasion du vote du rapport, l'avis minoritaire reste un "discours monogéré », puisqu'il ne pourra pas donner l'occasion à la réplique des autres eurodéputés, et que par ce fait « le locuteur a toute latitude d'élaborer ses stratégies discursives et de construire son ethos au gré de ce qu'il considère comme approprié à son objectif» (Amossy $2010: 219$ ). Dans ce cadre qui exclut la confrontation directe, nous voulons analyser de quelle manière l'expression de l'opinion minoritaire peut néanmoins donner lieu à un « mode polémique », entendu 
comme «mode verbal de gestion des conflits qui met l'accent sur l'antagonisme et l'incommensurabilité des points de vue opposés » (Amossy 2011).

\section{Types d'opinion minoritaire entre anti-discours et contre-discours}

9 Pour mieux comprendre le rôle de l'opinion minoritaire dans le cadre des rapports parlementaires non législatifs, partons du constat que si la visée du discours polémique est de « convertir » et de " vaincre » (Orkibi $2015: 10$ ), l'opinion minoritaire ne peut pas être considérée comme un discours polémique prototypique. En effet, à la différence du débat parlementaire où l'échange dialogal permet aux différents univers symboliques de s'affronter (Cabasino 2001: 104) et de négocier un large consensus sur la base de fondements communs avant d'en arriver au texte final, l'avis présenté au moment du vote démontre la persistance d'un dissensus qui ne peut plus se résorber. Il peut seulement se donner à voir dans le cadre de ce qui semble rester une pure «fiction polémique " où « les contradicteurs, afin de respecter le régime d'attentes attaché au jeu, au pacte polémique, s'appliquent donc conventionnellement à accentuer leurs dissensions - lesquelles restent bien réelles, il ne s'agit pas d'en douter -, et s'engagent à faire comme si rien ne pourrait jamais leur être commun, comme si tout les opposait, comme si leur désaccord était décidément indissoluble (Albert et Nicolas 2010 : 36).

Nous sommes alors en présence d'un «mode polémique » (Amossy 2011) dans la mesure où, comme nous le verrons par les exemples analysés dans les paragraphes suivants :

- il marque un contre-discours antagoniste qui vise la délégitimation de la vision du politique que se fait l'adversaire, en arrivant même, parfois, à frôler la violence verbale ;

- il essaie par tout moyen de disqualifier l'adversaire posé en simulacre dans le discours ;

- il polarise l'opposition « nous »-« eux », lors de la présence d'un collectif.

11 Il faut pourtant préciser qu'on peut trouver une différence fondamentale qui se fait jour lorsque sont utilisés des verbes régis par le pronom de la première personne du singulier ( «je »), dans le cas de la modalité individuelle, ou par la première personne plurielle ("nous »), lorsqu'apparaît une modalité polémique collective. Ces modalités caractérisent justement les deux types d'opinion minoritaire, «individuelle» et « collective », que nous avons trouvés dans notre corpus.

Essayons d'en cerner de près la spécificité par le tableau 1, où nous avons rapporté les verbes utilisés par les énonciateurs des opinions en question :

\begin{tabular}{|l|l|}
\hline JE & NOUS \\
\hline Réfutation & \\
- Je rejette (3 occurrences) & Réfutation \\
- Je désapprouve & - Nous nous opposons (8 occurrences) \\
- Je refuse & \\
\hline
\end{tabular}




\begin{tabular}{|l|l|}
\hline Rejet & Demande \\
- Je n'ai pas pu voter & Nous réclamons (2 occurrences) \\
- Je ne peux cautionner rien de tout cela & Nous appelons de nos vœux \\
& Nous avons besoin \\
\hline
\end{tabular}

Tableau 1 : Les verbes du contre-discours dans l'opinion individuelle (« je ») ou collective (« nous »)

13 Le tableau 1 montre que le rôle de l'opinion individuelle se réduit à la seule réfutation, ce qui amène l'eurodéputé en question à ne pas voter le rapport en se limitant à la contestation pure et simple, sans produire d'alternatives possibles. En revanche, lors de l'avis collectif, la réfutation est suivie par une proposition alternative qu'on défend haut et fort. Cette proposition s'accompagne d'arguments plus ou moins explicites, qui le plus souvent sont de communauté ${ }^{1}$ dans le sens où ils s'appuient sur des valeurs censées être partagées par la communauté discursive. Elles renvoient à une éthique démocratique, sociale et pacifiste largement partagée et de ce fait difficilement contestable («le désarmement radical [...] et le transfert des fonds des budgets militaires vers le budget social et du développement»; «un nouvel accord de coopération pacifique avec la Russie pour mettre fin aux tensions »; « la primauté des institutions politiques sur les institutions techniques, ainsi qu'une participation pleine et entière des parlements nationaux»). Plus rarement, ce sont des arguments de cadrage (par exemple, le cadrage de la Russie comme «un partenaire clé de l'Union européenne et un acteur essentiel au niveau mondial » plutôt que comme une menace, dans le cas du rapport Fotyga) ou d'autorité (l'accent mis et réitéré plusieurs fois, sur le fait que l'art. 41 du traité de l'Union européenne interdit d'utiliser le budget de l'Union pour les dépenses découlant des actions militaires ou de défense; le renvoi à d'autres articles du même traité).

14 Les deux types d'opinions minoritaires, individuelles et collectives, semblent donc donner lieu à deux contre-discours différents : si les premières se limitent à la simple réfutation du rapport en question en une sorte d'anti-discours, les deuxièmes offrent un discours «alternatif» (Rabatel 2015: 70) qui ne se contente pas de s'opposer au rapport de manière nette mais propose des choix différents et de véritables « trajectoires argumentatives possibles» (Doury, Quet, Tseronis $2015: 56$ ).

15 Cela dit, soulignons qu'en aucun cas - qu'il s'agisse d'opinion individuelle ou collective - il n'est fait mention de concessions à la partie adverse. Dans tous les cas analysés, les opinions minoritaires se caractérisent par la création en discours d'un contre-ethos qui oppose des valeurs et des images décalées par rapport à celles qui sont attribuées au rapporteur, et en général à l'adversaire politique, inscrit en simulacre dans le contrediscours. En effet, le dissensus ne s'estompe pas sur la base d'un ethos communautaire, bien au contraire, et cela à la différence de ce que laisseraient supposer les réflexions de Bendjaballah (2014: 72) qui parle d'une «Europe 'protectrice du citoyen'» comme référence partagée des députés dans le discours normatif du PE.

Ce sont justement ces modalités spécifiques de la polémique que nous allons analyser de plus près à l'aide des deux exemples sélectionnés, le premier concernant les opinions minoritaires parues dans les rapports sur la défense et la sécurité telles qu'elles ont été discutées par la commission des affaires étrangères pendant la législature actuelle du PE, le second ayant trait aux avis présentés dans les débats 
parlementaires sur la prostitution et sur l'égalité entre les hommes et les femmes telles qu'elles ont été abordées par la commission des droits de la femme et de l'égalité des genres à partir de la fin de législature précédente du PE et jusqu'à aujourd'hui.

\section{Opinions minoritaires dans les rapports ayant affaire à la défense et à la sécurité}

17 Dans le domaine de la défense, la nouvelle législature parlementaire est très active à la suite des problèmes soulevés par le terrorisme international. Dans ce cadre, la commission des affaires étrangères est sollicitée à plusieurs reprises et l'argument de la sécurité devient même un prétexte pour réformer la structure institutionnelle de l'Union, comme le démontre le rapport suivant produit par la commission des affaires constitutionnelles qui

souligne qu'outre le renforcement de la coordination entre les autorités et agences compétentes dans les États membres, Europol et Eurojust doivent obtenir de véritables compétences et capacités d'enquête et de poursuites, éventuellement par leur transformation en un véritable service européen de renseignement et de lutte contre le terrorisme, soumis à un contrôle parlementaire adéquat [...]

[et] note que ce n'est qu'en renforçant la politique étrangère et de sécurité commune que l'Union européenne peut apporter des solutions réalistes aux défis et menaces émergents en matière de sécurité, et ainsi lutter contre le terrorisme et restaurer la paix, la stabilité et l'ordre dans les pays de son voisinage [...].

Rapporteur : Guy Verhofstadt (20/12/2016)

18 C'est précisément par rapport à la politique étrangère et de sécurité ainsi définie que les opinions minoritaires se prononcent.

\subsection{La réfutation passe par des « routines »}

Quatre des cinq opinions minoritaires présentes dans les rapports sur la défense et la sécurité montrent la présence d'une véritable "routine " ${ }^{2}$ (Née, Sitri, Véniard 2016 : 72-76) d'opposition polémique, d'après le schéma triadique suivant :

1. «Le rapport demande / recense / affirme » $+\mathrm{X}$

2. «Nous nous opposons à ce rapport» + "pour les raisons suivantes / dans la mesure où il :

3. « Nous »+ « demandons / réclamons »+ X.

Dans la routine, le positionnement de contestation ne se fait pas par rapport à des individus, la critique concernant directement le rapport, qui devient dès lors l'objet de l'opposition («nous nous opposons à ce rapport»). Il est donc intéressant de relever que les énonciateurs posent leurs revendications à travers un «nous» pluriel, qui les désigne à la fois en tant que membres du groupe politique qu'ils représentent et en tant que porte-paroles de ce même groupe. Qui plus est, ils font exister ce groupe par la polémique contestataire de la revendication collective. Nous sommes donc en présence d'un type d'opinion « collective ».

21 La routine triadique révèle également la présence de véritables " éléments de langage » (Ollivier-Yaniv 2011 : 65 ; Krieg-Planque, Oger 2017), c'est-à-dire d'éléments stables qui sclérosent le discours et renvoient à la présence de pratiques normatives « contribu[a]nt à cadrer les débats et à faire circuler sous une forme stabilisée les positionnements des instances gouvernantes» (Bonhomme et Devrient-Monte, cités 
dans Krieg-Planque, Oger 2017). C'est par exemple ce qui arrive dans le cas présenté dans le tableau 2, où certains segments du rapport sur l'UE de la défense, présenté par Urma Paet en octobre 2016, sont repris tels quels dans le rapport annuel de mise en œuvre de la politique de la sécurité et de défense commune signé par Michael Gahler en 2017 :

\begin{tabular}{|c|c|}
\hline $\begin{array}{l}\text { Rapport sur l'Union européenne de la défense } \\
(31 / 10 / 2016) \\
\text { Rapporteur : Urmas Paet }\end{array}$ & $\begin{array}{l}\text { Rapport sur le rapport annuel sur la mise en } \\
\text { œuvre de la politique de sécurité et de défense } \\
\text { commune } \\
(31 / 12 / 2017) \\
\text { Rapporteur : Michael Gahler }\end{array}$ \\
\hline $\begin{array}{l}\text { Opinion minoritaire exprimée par les députés GUE/NGL } \\
\text { Sabine Lösing et Takis Hadjigeorgiou }\end{array}$ & $\begin{array}{l}\text { Rapport minoritaire déposé par les députés GUE/ } \\
\text { NGL Sabine Lösing, Takis Hadjigeorgiou, Miguel } \\
\text { Urban Crespo, Javier Couso Permuy }\end{array}$ \\
\hline $\begin{array}{l}\text { Le rapport demande instamment la création d'une } \\
\text { Union militaire dotée de capacités militaires } \\
\underline{\text { fortes. Il demande l'activation... }}\end{array}$ & $\begin{array}{l}\text { Le rapport demande instamment la création } \\
\text { d'une Union militaire dotée de capacités } \\
\text { militaires fortes. Il soutient la création d'un } \\
\text { fonds européen de la défense }\end{array}$ \\
\hline $\begin{array}{l}\text { Nous nous opposons à ce rapport pour les raisons } \\
\text { suivantes: } \\
\text { il réclame au minimum } 90 \text { millions d'euros pour } \\
\text { des actions préparatoires dans la recherche en } \\
\text { matière de défense et un programme de recherche } \\
\text { dans le domaine de la défense entièrement financé } \\
\text { par l'Union de } 500 \text { millions d'euros par an à partir } \\
\text { de } 2021 \text {; }\end{array}$ & $\begin{array}{l}\text { Nous nous opposons à ce rapport dans la mesure } \\
\text { où il: } \\
\text { invite à renforcer la coopération entre l'Union } \\
\text { et l'OTAN et le mandat des missions de la } \\
\text { PSDC; }\end{array}$ \\
\hline $\begin{array}{l}\text { Nous réclamons: } \\
\text { - l'interprétation stricte de l'article 41, } \\
\text { paragraphe } 2, \text { du traité UE qui interdit d'utiliser le } \\
\text { budget de l'Union pour les dénenses découlant des }\end{array}$ & $\begin{array}{l}\text { Nous demandons: } \\
\text { - l'interprétation stricte de l'article 41, } \\
\text { paragraphe 2, du traité UE qui interdit }\end{array}$ \\
\hline actions ayant des implications militaires ou de & dépenses découlant des actions ayant des \\
\hline $\begin{array}{l}\text { défense; } \\
\text { - le désarmement radical (y compris CBRN) au } \\
\text { niveau de l'Union et de la planète et le transfert }\end{array}$ & $\begin{array}{l}\text { implications militaires ou de défense; } \\
\text { - la cessation des financements militaires par } \\
\text { le budget de l'Union; le désarmement radical }\end{array}$ \\
\hline $\begin{array}{l}\text { des fonds des budgets militaires vers le budget } \\
\text { social et du développement; }\end{array}$ & $\begin{array}{l}\text { (y compris CBRN) au niveau de l'Union et de la } \\
\text { planète et le transfert des fonds des budgets }\end{array}$ \\
\hline $\begin{array}{l}\text { - la cessation des financements militaires par le } \\
\text { budget de l'Union. }\end{array}$ & $\begin{array}{l}\text { militaires vers le budget social et du } \\
\text { développement. }\end{array}$ \\
\hline
\end{tabular}

Tableau 2 : Reprise du dit (en souligné) et utilisation de routines (en italiques) dans les opinions minoritaires concernant la défense et la sécurité

D'une part, la présence d'éléments préfabriqués que les énonciateurs répètent tels quels, à quelques détails près, finit par standardiser le discours polémique, mais de l'autre, elle facilite la reprise d'arguments qui peuvent être aisément isolés, détachés et réutilisés dans d'autres contextes. Les éléments de langage agissent ainsi également sur 
la réception du discours (Krieg-Planque, Oger 2017) en ce qu'ils mettent à disposition des éléments préconstruits - des «briques » (segments de textes, arguments...) - qu'on reprend sans variation et qui sont conçus pour susciter certains effets dans l'auditoire.

Il n'en va pas toujours ainsi, comme le démontre le seul avis minoritaire qui semble échapper à la routine, le cinquième. Cet avis, en effet, se structure différemment : il ne présente pas de routine triadique et se limite à avancer au début la structure phrastique de l'opposition, cette fois-ci rapportée à la troisième personne : «Pour des raisons de principe, le groupe ENF s'oppose à ce rapport ». L'absence de figement du discours lui confère une authenticité et une imprévisibilité majeures. En outre, remarquons que, dans cet avis, le positionnement ne s'effectue pas vraiment en relation au rapport mais plutôt à l'égard du rapporteur, en une sorte d'argument ad hominem, ou mieux ad personam (Viktorovich 2010 : 95), qui s'en prend directement à l'adversaire et à son ethos de crédibilité (« s'entête dans un dogme [...] partisan déclaré d'une [...] idée et une orientation [...] contraires à l'identité européenne ») :

Le rapporteur s'entête totalement dans le dogme d'une logique d'une Union qui ne cesse de s'étendre et de s'approfondir. Il est également un partisan déclaré des "États-Unis d'Europe », une idée et une orientation qui sont à nos yeux contraires à l'identité européenne, laquelle doit se fonder sur la primauté et la valeur de l'identité nationale.

On remarque qu'en réalité, cet argument est à la fois personnel et circonstanciel (Gauthier 1995: 174-175). D'une part, il vise la délégitimation de l'adversaire, en laissant entendre qu'il est têtu, voire déraisonnable et dogmatique. D'autre part, il travaille à délégitimer le positionnement du rapporteur par la redéfinition de l'identité européenne, légitimant en retour l'énonciateur lui-même et ses valeurs, ce qui contribue à redéfinir les valeurs communes et les règles de la rationalité qu'il faut partager dans l'espace public (Oger $2012: 3$ ).

À la différence des quatre autres avis précédemment analysés, celui-ci est prononcé par un seul rapporteur, Gerolf Annemans qui le présente au nom de son groupe; dans ce sens, son type de contestation, directement adressée aux acteurs politiques, s'apparente aux opinions minoritaires «individuelles » que nous analyserons plus bas, en étudiant le cas des opinions sur l'égalité de la commission des droits de la femme et de l'égalité des genres.

26 À l'exception donc de ce dernier cas, les avis minoritaires de la commission des affaires étrangères cités montrent la tendance des opinions collectives à se figer dans des structures discursives spécifiques (routines et éléments préfabriqués), ce qui leur permet de véhiculer la polémique dans les formes discursives qui caractérisent de façon générale le discours institutionnel (Krieg-Planque 2012 : 81).

\subsection{Des valeurs et des ethè opposés}

27 Si la structure triadique et la présence de segments qui se répètent tendent à figer le discours de l'opinion minoritaire collective, il importe d'examiner de plus près comment le mode polémique ressort de ce discours. C'est ce que nous allons faire à l'aide du tableau 3 : 


\section{4/10/2016 (rapport Fotyga) \\ Opinion minoritaire du groupe GUE/NGL}

Nous nous opposons à ce rapport pour les raisons suivantes :

- il est irresponsable de placer un État comme la Russie au même niveau de menace de Daech ;

- il encourage la course de l'Union aux accords de libre-échange avec des pays du voisinage oriental et au-delà, favorisant ainsi une escalade des tensions avec la Russie et son union économique eurasiatique ;

- il ne fait pas apparaître le rôle négatif et croissant de l'Union dans les conflits qui touchent actuellement son voisinage méridional et oriental ni la propagande élaborée, principalement par les États-Unis, pour légitimer ses interventions passées, par exemple en Iraq, qui ont semé le chaos dans la région;

- il ne reconnaît pas sa propre propagande sur la démocratie de l'Union qu'il présente comme la seule forme possible de démocratie, ce qui fait montre d'une prétendue supériorité qui pourrait être offensante ;

- il recommande paradoxalement que les « médias indépendants » soient soutenus par l'Union.

31/10/2016 (rapport Paet)

Opinion minoritaire des députés GUE/NGL :

Ce rapport encourage [...] exige des États membres qu'ils œuvrent à atteindre l'objectif de $2 \%$ du PIB fixé pour les dépenses de défense.

Nous réclamons :

- l'interprétation stricte de l'article 41, paragraphe 2, du traité UE qui interdit d'utiliser le budget de l'Union pour les dépenses découlant des actions ayant des implications militaires ou de défense ;

08/12/2016 (rapport Verhofstadt)

Opinion minoritaire des députés CER :

L'incapacité de l'Union européenne à remédier efficacement aux crises auxquelles elle est confrontée démontre qu'elle est allée trop loin. Elle est trop centralisée et éloignée de ses citoyens. Elle en fait trop, et elle fait mal. Les récents référendums et élections montrent que le public est de plus en plus sceptique quant à la valeur de l'Union, aux objectifs qu'elle poursuit et à sa capacité de produire des résultats.

Opinion minoritaire des députés ENF :

Ce n'est qu'en mettant un terme à cette agressivité vis-à-vis de l'existence des États membres et en restaurant la souveraineté nationale que nous pourrons prévenir d'autres dégâts. Ce n'est que de cette façon que l'on pourra respecter les résultats de nombreux référendums ignorés ou discrédités sur l'avenir de l'Europe.

av13/11/2017 (rapport Gahler)

Opinion minoritaire des députés GUE/NGL :

Nous nous opposons à ce rapport dans la mesure où il :

il exige des États membres qu'ils augmentent les dépenses de la défense [...]

Nous demandons :

- l'interprétation stricte de l'article 41, paragraphe 2, du traité UE qui interdit d'utiliser le budget de l'Union pour les dépenses découlant des actions ayant des implications militaires ou de défense ; 
13/11/2017 (rapport McAllister)

Opinion minoritaire des députés GUE/NGL :

Nous nous opposons pour les raisons suivantes :

- il parle de résolution pacifique et diplomatique de conflits mais soutient une industrie de la défense forte et demande d'augmenter les financements pour les moyens de défense ;

- il demande l'abolition du principe d'unanimité au Conseil concernant la PESC mais s'exprime en faveur des décisions à la majorité qualifiée [...]

Tableau 3 : La contestation dans les avis minoritaires concernant la défense et la sécurité

28 Le tableau 3 montre au moins trois stratégies discursives et lexicales de contestation polémique que nous allons aborder en détail plus bas :

1. la contestation peut jouer sur des implicites qui finissent par attribuer au rapporteur, inscrit dans les exemples de façon purement métonymique («le rapport»), un ethos anti-européen au sens où l'énonciateur entend la vraie Europe de manière différente, tout en s'appuyant sur les principes et les documents fondateurs de l'UE ;

2. la création de groupes opposés: le "nous» du groupe minoritaire oppose son éthique à celle du simulacre de l'adversaire politique (le rapporteur et ceux qui approuvent son rapport) ;

3. la valeur argumentative de certains mots (Amossy 2010: 138), notamment de certaines dénominations qui positionnent l'énonciateur dans l'interdiscours, comme c'est le cas de « Daech ».

Par rapport à l'utilisation des implicites, on note que c'est souvent par eux que passe le processus de délégitimation du rapporteur. L'énoncé « il est irresponsable de placer un État comme la Russie au même niveau de menace de Daech » présuppose que, dans le rapport en question, la Russie et Daech ont été mis au même plan, ce qui produit un acte «irresponsable» de la part du rapporteur, qui ne serait donc pas capable d'attribuer aux choses leur juste valeur. La même crédibilité fait défaut dans l'énoncé « il [le rapport] recommande paradoxalement que les "médias indépendants" soient soutenus par l'Union", ce qui implique une forme de contradiction interne. Il en va de même, enfin, pour le cas où les contestataires s'appuient sur l'«interprétation stricte de l'article 41, paragraphe 2, du traité UE qui interdit d'utiliser le budget de l'Union pour les dépenses découlant des actions ayant des implications militaires ou de défense ", ce qui renvoie à l'implicite selon lequel le rapporteur, prévoyant des dépenses de défense, en vient à violer l'un des principes de l'UE.

Le rapporteur-simulacre, qui est inscrit dans le discours, est donc quelqu'un d'irresponsable, voire d'« agressif ", comme le laisse entendre le rapport Verhofstadt en tableau 3, où les députés souhaitent justement qu'on mette "un terme à cette agressivité vis-à-vis de l'existence des États membres ", ce qui par un raisonnement métonymique implicite dénonce, d'une part, l'agressivité des politiques menées par l'UE aux dépens de la souveraineté des États membres, et d'autre part, des groupes politiques majoritaires (dont celui du rapporteur et, de ce fait, le rapporteur lui-même) qui la soutiennent.

31 Le rapporteur est donc censé être incapable de bien décider de l'avenir européen et d'écouter les peuples. C'est justement par rapport à ces capacités que se joue la dissociation «nous»-«eux». S'il est vrai que, dans les discours du PE, ce couple 
d'opposés finit normalement par caractériser le «nous» des Européens contre le « eux » des non-Européens (Kriszan 2011, Carta 2015), dans les opinions minoritaires par contre, le "nous" éclate de l'intérieur pour manifester la présence de deux groupes opposés : ceux qui ont l'Europe à cœur contre ceux dont les actions dénotent l'absence d'une "vraie" éthique européenne. En ce sens, le discours de l'opinion minoritaire est fondamentalement polémique dans la mesure où il entend " polariser le monde entre "juste" et "erroné", entre "nous" et "eux" (Garand 2007: 14-16), et "discréditer des adversaires sur la base de leurs personnalités et de leurs positions " (Orkibi $2015:$ 10).

Dans ce mode polémique, le « nous » des opposants-contestataires se légitime :

- par le «parler vrai » (Le Bart 2014 : 40-41), là où le rapporteur en vient à ne pas dire les choses telles qu'elles sont vraiment. Dans les énoncés suivants, le manque de transparence est dénoncé par le «mais» adversatif: le rapport "parle de résolution pacifique et diplomatique de conflits mais soutient une industrie de la défense forte et demande d'augmenter les financements pour les moyens de défense; il demande l'abolition du principe d'unanimité au Conseil concernant la PESC mais s'exprime en faveur des décisions à la majorité qualifiée ». Ce type de "mais » finit par opposer les deux parties des énoncés en soulignant l'incohérence entre les effets d'annonce et les pratiques concrètes de l'adversaire politique, en une sorte d'argument ad hominem circonstanciel (Gauthier 1995: 174) qui délégitime le rapporteur et contribue à légitimer les contestataires en tant que véritables défenseurs des principes concernés et d'un langage qui ne se réduit pas aux seuls effets d'annonce ;

- par la dénonciation des échecs mêmes d'une Europe qui n'est plus en mesure de donner de vraies réponses, comme le montrent les axiologies négatives (« Elle est trop centralisée et éloignée de ses citoyens. Elle en fait trop, et elle fait mal»; «il [le rapport] ne fait pas apparaitre le rôle négatif et croissant de l'Union dans les conflits qui touchent actuellement son voisinage méridional et oriental »). La dénonciation finit par légitimer les contestataires en ce qu'ils proposent par les moyens de l'implicite une vision alternative qui est posée comme positive: la demande d'une Europe qui soit proche des citoyens et qui change de rôle au plan international.

Cette opposition de valeurs (responsabilité vs irresponsabilité ; souveraineté nationale vs destruction des États membres; violation du droit européen vs respect des principes et des articles européens; imposition de ses propres points de vue et tendance à favoriser les tensions $v s$ tolérance et paix) manifeste la présence d'un opposant, ici collectif, qui construit son contre-ethos en discours ${ }^{3}$. Ce contre-ethos sous-entend l'existence d'imaginaires discursifs opposés, la souveraineté nationale caractérisant la polémique des députés de droite (ENF), le désarmement et la paix marquant les priorités de la gauche (GUE/NGL).

L'opposant fait également des choix précis au niveau lexical pour marquer ses divergences face à l'adversaire politique, en attribuant à certains mots une valeur argumentative. C'est justement ce qui arrive lors de l'utilisation de «Daech» dans le premier avis minoritaire du tableau 3. Il est à relever dans la mesure où normalement, dans les rapports du PE, le terme qui devrait être utilisé est le couple «EIIL/Daech », ce dernier étant le terme recommandé par la banque des données terminologiques de l'UE, IATE. Le remplacement de l'expression par le plus simple «Daech » semble alors marquer le positionnement du groupe concerné qui privilégie l'expression péjorative, normalement utilisée par le Gouvernement français depuis 2014, par rapport au couple 
où paraît également l'EIIL, qui est notamment utilisé par le Conseil de sécurité de l'ONU comme forme abrégée de l'État islamique en Iraq et au Levant. Par ce choix, l'énonciateur espagnol Javier Couso, porte-parole du groupe en question, marque une prise de position collective critique vis-à-vis du rapporteur, qui privilégie le couple «EIIL/ Daech ». De cette manière, Couso s'aligne sur des positionnements plus « autonomes » comme celui des Français.

Nous allons examiner de plus près en quoi consiste cette procédure discursive en nous penchant sur le cas de la dénomination des objets dans les rapports de la commission des droits de la femme et de l'égalité des genres.

\section{Opinions minoritaires dans les rapports de la commission des droits de la femme et de l'égalité des genres}

Notre deuxième cas d'analyse porte sur les avis minoritaires qui paraissent dans les rapports produits par la commission des droits de la femme et de l'égalité des genres à partir de la fin de la septième législature et jusqu'à aujourd'hui. Ces opinions nous permettront non seulement d'observer en détail la dénomination polémique des objets, mais également d'observer les modalités polémiques des avis individuels.

Nous commencerons par l'analyse du rapport sur l'exploitation sexuelle et la prostitution, et leurs conséquences sur l'égalité entre hommes et femmes qui clôt le débat européen sur la prostitution le 3 février 2014. Ce rapport, signé par l'anglaise Mary Honeyball, est fondamental dans la mesure où, par l'interdiction de toute forme de prostitution, il marque la fin de la confrontation entre le positionnement réglementariste, visant la condamnation de la seule prostitution forcée, et le positionnement abolitionniste, qui souhaitait l'abolition de toute forme de prostitution. C'est justement par rapport à la décision finale d'interdire la prostitution sous toutes ses formes que les opinions minoritaires sont présentées : d'une part, celle d'Angelika Niebler, Christa Klaß et Astrid Lulling et de l'autre, celle d'Ulrike Lunacek, Marije Cornelissen, Iñaki Irazabalbeitia Fernández, Raül Romeva i Rueda et Sophia in 't Veld. Nous allons tout d'abord analyser ces deux avis pour ensuite aborder le cas de ceux qui concernent de façon plus générale l'égalité entre les femmes et les hommes dans la législature en cours.

\subsection{La lutte politique passe par la dénomination des objets}

Dans le cas de la prostitution, le positionnement des avis minoritaires semble confirmer les stratégies déjà relevées dans l'analyse des rapports sur la sécurité et la défense. La position de Niebler, Klaß et Lulling commence par ces termes : «Le présent rapport d'initiative entend interdire toute forme de prostitution. Toutefois, nous estimons qu'il faut faire une distinction entre la prostitution forcée et la prostitution légale, comme le prévoit la législation communautaire ».

Le positionnement par rapport au rapporteur-simulacre se fait par un "nous " qui s'appuie sur les principes et/ou la législation de l'UE pour délégitimer l'adversaire (" comme prévoit la législation communautaire »). L'inscription de l'adversaire se fait toujours par référence au « rapport »: « Le rapport de Mary Honeyball comporte des 
propositions utiles pour les États membres en ce qui concerne les questions liées à la prostitution forcée ».

40 C'est par le paradigme désignationnel (Mortureux 2004: 100) "prostitution légale", «prostitution volontaire » que les énonciatrices reformulent l'objet qui, d'après leurs propres valeurs, appelle la politique qu'elles proposent, et qu'elles naturalisent par la modalité déontique: "La façon de traiter la prostitution volontaire doit rester du ressort de chaque État membre. [...] La prostitution forcée et la traite des êtres humains doivent être combattues par tous les moyens disponibles ».

Dans le deuxième avis minoritaire sur la prostitution, il est question des dénominations, comme on peut le voir par la modalité autonymique suivante :

La traite d'êtres humains, notamment des femmes et d'enfants, à des fins d'exploitation sexuelle, qu'on appelle également "prostitution forcée ", constitue une violation de la dignité humaine et est contraire aux principes de la charte des droits fondamentaux de l'Union européenne.

Cette fois-ci le collectif préfère désigner la prostitution forcée comme une «traite d'êtres humains ", ce qui marque tout de suite le positionnement des eurodéputés concernés. Dans l'extrait, les locuteurs s'appuient sur les valeurs européennes de la Charte, le positionnement vis-à-vis du rapport s'effectuant une fois de plus par le recours à l'éthique et par les dénominations des objets: «le rapport ne fait pas de distinction entre prostitution forcée et prostitution résultant d'une décision individuelle ».

Si maintenant nous passons aux opinions concernant l'égalité entre les femmes et les hommes dans la législature en cours, nous assistons à une véritable «lutte terminologique » dans l'avis minoritaire présenté par Anna Záborská en juin 2015 :

le texte adopté par la commission donne délibérément la préférence au terme juridiquement ambigu de "gender » plutôt qu'au mot «sex», quant à lui clairement défini, et gomme ainsi la distinction entre les deux sphères de l'existence humaine : sociale et privée. Le terme équivoque de "gender » laisse la porte ouverte à des interprétations erronées. Dès lors, l'iniquité et l'intervention de l'État dans la sphère privée des citoyens européens prévaudront sur la justice. C'est pourquoi je n'ai pas pu voter en faveur de ce texte.

Par les commentaires métalinguistiques, Záborská laisse entendre que la commission parlementaire adopte un positionnement précis par rapport aux termes concernés et au flou conceptuel du terme "gender» («la commission donne délibérément la préférence au terme juridiquement ambigu»), dont l'interprétation entraînerait des dérives dangereuses auxquelles la rapporteuse s'oppose en ne votant pas son propre texte ${ }^{4}$. Dans l'avis de Záborská, le mode polémique s'exprime dans l'attribution aux termes d'une valeur argumentative d'orientation qui affecte le dit («[1]e terme équivoque de "gender " laisse la porte ouverte à des interprétations erronées ») et l'action du PE et, par conséquent, des États membres («l'iniquité et l'intervention de l'État dans la sphère privée des citoyens européens prévaudront sur la justice »).

La dénomination de l'objet n'est donc pas simplement un problème de positionnement au plan de l'interdiscours; c'est d'elle que dépend l'action des politiques européennes dans la mesure où tout terme renvoie à un concept juridique opératoire. On retrouve un cas similaire lors de la contestation de Beatrix Von Storch du 10 février 2016: "Aucune différence n'est faite entre les réfugiés fuyant les guerres et les réfugiés économiques. Les États membres n'auraient pas le droit de contrôler leurs propres frontières nationales. Je ne peux cautionner rien de tout cela ». L'exemple porte donc 
sur la clarté conceptuelle et le manque de "distinction » opératoire des termes qui avaient déjà caractérisé le débat sur la prostitution.

\subsection{L'opinion « individuelle »}

Contrairement aux opinions précédentes, qui pour la majorité étaient présentées par plusieurs eurodéputés, ce qui permettait l'utilisation du «nous » pluriel se confondant avec le «nous » de porte-parole, l'avis de Záborská et celui de Von Storch que nous venons de citer sont le fruit d'une opinion personnelle, ce qui permet l'utilisation du «je».

Le cas de Von Storch est particulièrement intéressant parce que cette députée allemande est l'auteure de la moitié des opinions minoritaires présentes dans les rapports de la commission des droits de la femme et de l'égalité des genres. Par cette tendance à présenter fréquemment des opinions minoritaires, nous pouvons apparenter le «profil » de cette eurodéputée au personnage du « contestataire », dont l'anticonformisme se caractérise justement par «la dénonciation récurrente et systématique du mode de fonctionnement du Parlement européen, la prise de distance à l'égard de ses résolutions elle-même et la défiance envers les autres signataires " (Navarro $2009: 501)^{5}$.

8 L'expression du dissensus recouvre dans ses avis plusieurs modalités, mais celles-ci concourent toutes à contester la doxa européenne par des arguments de cadrage :

(02/08/2016) L'UE considère au premier chef les femmes comme des travailleuses. En vertu de l'agenda 2020, 75 \% d'entre elles doivent être intégrées au marché du travail formel. La liberté de choix est expressément non souhaitée. Ces mêmes femmes (en âge de procréer) sont considérées, pour surmonter la crise démographique, en tant que mères. Le même groupe cible fait l'objet de deux approches politiques inconciliables. La grande perdante, c'est la famille. Le traité FUE ne connaît en effet que des travailleurs, et non des pères et des mères. Malgré cela, l'UE veut régir la vie de famille et définir les valeurs et les normes en son sein. [...] Il n'y est nullement question de famille ni de parentalité, mais de la compatibilité de toutes les femmes au marché du travail. [...] Je désapprouve cette vision des choses.

La désapprobation passe par la dénonciation du stéréotype de la femme-mèretravailleuse, qui caractérise le discours néolibéral de l'UE (Rizzitelli 2012), ce qui permet aussi la dénonciation d'une éthique antifamiliale et délégitime l'adversaire politique.

La stratégie de délégitimation de la partie adverse est d'ailleurs la plus répandue dans les interventions de l'eurodéputée allemande, qui vont jusqu'à la violence verbale ${ }^{6}$ :

(13/05/2015) Ce rapport exige entre autres la reconnaissance de l'avortement comme un droit de l'homme. [...] d'après le droit pénal allemand en vigueur, l'avortement constitue une infraction [...] Sur le fond, je rejette ces programmes de sexualisation et, sur le plan juridique, la politique éducative relève également des compétences des États membres. Ce ne sont que quelques-uns des motifs me poussant à rejeter ce rapport. Il y en a de nombreux autres...

(10/02/2016) Angela Merkel (CD/PPE) porte un préjudice sans précédent à l'Union européenne depuis 1945. Par la décision qu'elle a prise en cavalier seul en tant que chancelière, elle a enfreint le droit constitutionnel et le droit européen et a favorisé l'émergence de la crise des réfugiés. Sa démarche volontariste empiète sur les compétences techniques de la commission des affaires intérieures, occasionne une 
charge supplémentaire considérable pour les aidants bénévoles, les conseillers des Länder, contredit le principe fondateur de l'égalité devant la loi

(12/05/2017) Ce rapport n'est qu'un autre exemple de gesticulation parlementaire. [...] L'Union européenne nie la liberté de choix des pères et des mères de famille. Elle vise uniquement à concrétiser les objectifs de Barcelone sur la mise en place de services publics de garde d'enfants. L'Union nie ainsi délibérément la liberté de choix des parents qui souhaitent élever leurs enfants selon des modèles différents. [...] Le véritable scandale ne réside pas tant dans de prétendus écarts de salaire ou de pension que dans le manque de reconnaissance du travail fourni de manière égale par les femmes et les hommes.

51 Des mots comme "préjudice ", " empiète ", " gesticulation ", "scandale " sont des marqueurs de la volonté de discréditer l'adversaire, Angela Merkel, qui d'ailleurs est également délégitimée par l'argument ad hominem personnel (« en cavalier seul »). Mais ils discréditent aussi l'Europe telle qu'elle est actuellement ; la posture de l'énonciatrice frôle souvent l'euroscepticisme. L'Union et ses représentants se voient attribuer une image de véritables « hors-la-loi » dans la mesure où ils sont accusés de commettre une infraction par l'interdiction de l'avortement, de contredire le principe de l'égalité devant la loi, de nier la liberté de choix des parents - l'argumentation périphérique étant le plus souvent circonstancielle (Gauthier 1995).

Remarquons que la contestation selon laquelle l'UE tendrait à limiter les compétences des États membres par une politique trop agressive est fréquente dans les avis qui sont signés en solo mais aussi dans les opinions présentées en groupe, comme nous l'avons vu dans l'opinion des députés ENF dans le rapport Verhofstadt de décembre 2016 (voir tableau 3). Pour les contestations individuelles, outre la critique de Von Storch ( «la politique éducative relève également des compétences des États membres»), voici l'accusation de Marek Jurek :

(19/07/2017) l'aspiration de l'Union d'adhérer à cette convention [du Conseil de l'Europe] est un signe qu'elle souhaite s'immiscer dans les différends légitimes en cours à l'intérieur des États membres (y compris les plus anciens et les plus grands comme l'Allemagne). Il s'agit donc de la manifestation d'une passion idéologique qui dédaigne l'État de droit, et en particulier le principe d'attribution des compétences prévu à l'article 5 du traité sur l'Union européenne.

53 Ici, et de nouveau, il n'est plus question de s'en prendre au seul rapporteur, à la commission parlementaire ou à un adversaire spécifique (comme Angela Merkel), adversaire qui d'ailleurs, et à la différence de la situation dialogale (Albert, Nicolas 2010 : 28), ne pourra pas répondre aux sollicitations, mais de s'écarter en général des politiques actuelles de l'Union européenne qui risquent de violer les souverainetés nationales et même de porter atteinte à la législation européenne. En cela les arguments utilisés acquièrent une valeur circonstancielle - en tant qu'arguments « de tartuffe» (Gauthier 1995: 177) - dans la mesure où ils laissent entendre une incohérence entre la conformité aux règles européennes et les comportements et les politiques (le faire) adoptés par les acteurs contestés.

\section{Conclusion}

En conclusion, rappelons le constat fait par Amossy et Burger (2011), selon lequel la polémique est un phénomène qui « s'inscrit dans un espace dialogique au sein duquel il constitue 'un discours a contrario' [...] qui se focalise sur le discours de l'autre pour le rejeter ». En ce sens, comme nous l'avons vu, les opinions minoritaires relèvent du 
"mode polémique " au sens où elles opposent de manière presque manichéenne le discours du rapport et un discours contestataire réfutant la vision doxique d'une Europe protectrice et un ethos communautaire censé être partagé avec l'adversaire. Dénonciation d'un consensus "obligé " qui ne fait pas l'unanimité, ces opinions, reléguées au fond du rapport, ne peuvent cependant pas réorienter le dit majoritaire à l'intérieur du PE, qui finalement reste hégémonique.

Dans ce cadre, nous avons fait pourtant un distinguo entre les discours contestataires présentés par un seul eurodéputé, qui finissent par créer un anti-discours sans véritable alternative, et les opinions de groupe qui non seulement font exister des collectifs qui se légitiment et se donnent à voir par le contre-discours mais qui, tout en alimentant un discours alternatif dont la structure tend à se figer dans des routines proches de celle des manifestes ${ }^{8}$, contribuent à faire circuler des contre-arguments. Ces derniers ne sont pas uniquement donnés à voir aux collectifs antagonistes lors du vote au PE; ils seront également - et c'est le plus important - communiqués au «tiers » absent, notamment les commissaires européens et/ou les autres acteurs concernés dans l'espace public européen (Conseil de l'Europe, lobbies...) qui liront les rapports. Par conséquent, ces arguments pourront être repris et réutilisés pour finalement alimenter des discours alternatifs en dehors du PE, qui pourront à leur tour soit être réduits au silence, soit contribuer à rectifier le discours de cette institution (Raus 2015 : 118), les «tiers » pouvant proposer à leur tour des contre-discours que le PE sera plus au moins obligé de prendre en compte ${ }^{9}$. En cela, ce type d'avis permet aux commissions parlementaires européennes de jouer un rôle similaire à celui des réunions des commissions parlementaires françaises en tant qu'« espace de répétition, permettant aux représentants de roder leurs arguments » (Viktorovich $2010: 110$ ).

Cet aspect confirme l'importance prépondérante des groupes et des collectifs, qui tendent à devenir les porte-paroles du dissensus dans l'espace public européen, le rôle d'opposition des individus se limitant plutôt à la contestation pure et simple à travers l'argument ad hominem personnel, qui vise la délégitimation de l'adversaire, et l'argument ad hominem circonstanciel, qui sert à délégitimer le positionnement de l'adversaire. Remarquons que l'argument personnel semble convenir à la contestation de la droite nationaliste (Gerolf Annemans, Beatrix Von Storch), alors que l'argument circonstanciel est le plus utilisé, notamment par les groupes, toutes positions politiques confondues.

En effet, les groupes aussi ont tendance à utiliser l'argument ad hominem pour discréditer l'adversaire et son positionnement, mais leur contre-discours se veut en même temps alternatif dans la mesure où il propose un "contre-monde " (Carbou 2015 : 81), qui s'exprime par ses propres mots et arguments, tout en ayant tendance à les faire circuler dans des structures sclérosées (routines et éléments " préfabriqués »). Ce penchant de l'opinion minoritaire à évoluer vers un genre discursif "routinier " (Maingueneau $2007: 30$ ) en ce que, sous sa forme collective actuelle, elle tend à laisser moins de marge de variation et à figer la matérialité discursive dans des structures prévisibles, mérite sans doute des analyses ultérieures portant également sur les conditions de production de ces discours. En effet, il peut sembler paradoxal que, pour contester le discours majoritaire hégémonique, on finisse par recourir à des structures discursives fort proches de celles qui caractérisent le discours contesté. collectives qui permet aux opinions minoritaires actuelles de jouer un rôle fondamental 
dans le débat public en tant qu'elles finissent par contribuer à l'image d'une Europe entendue comme un "espace discursif » où les « discours et contre-discours circulent et se confrontent dans un espace qui existe à la fois par l'entente et par le conflit et, conjointement, contribuent à des constructions institutionnelles» (Auboussier \& Ramoneda 2015 : 10-11).

\section{BIBLIOGRAPHIE}

Albert, Luce \& Loïc Nicolas (éds). 2010. Polémique et rhétorique. De l'Antiquité à nos jours (Bruxelles : De Boeck)

Amossy, Ruth. 2010. L'argumentation dans le discours (Paris : Colin, $3^{\mathrm{e}}$ édition)

Amossy, Ruth. 2011. « La coexistence dans le dissensus. La polémique dans les forums de discussion ", Semen 31, 25-42

Amossy, Ruth. 2014. Apologie de la polémique (Paris : Presses Universitaires de France). Amossy Ruth \& Marcel Burger. 2011. «Introduction : la polémique médiatisée », Semen 31, 7-24

Amossy Ruth \& Roselyne Koren. 2010. « Argumentation et discours politique », Mots 94, 13-21 Auboussier, Julien \& Toni Ramoneda (éds). 2015. L'Europe en contre-discours (Besançon : Presses Universitaires de Franche-Comté)

Bendjaballah, Selma. 2014. «La pratique de la négociation dans une démocratie de consensus : le cas des députés du Parlement européen », Négociations 21, 65-78.

Breton, Philippe. 2015. Convaincre sans manipuler. Apprendre à argumenter (Paris : La Découverte)

Cabasino, Francesca. 2001. Formes et enjeux du débat public. Discours parlementaire et immigration (Rome : Bulzoni).

Carbou, Guillaume. 2015. «Des contre-discours aux contre-mondes : l'exemple des commentaires d'internautes autour de l'accident de Fukushima », Semen 39, 81-97.

Carta, Caterina. 2015. “The Swinging 'we'. Framing the European Union International Discourse”. Journal of Language and Politics 14-1, 65-86

Charaudeau, Patrick. 2005. Le discours politique. Les Masques du Pouvoir (Paris : Vuivert)

Doury Marianne, Mathieu Quet \& Assimakis Tseronis 2015. « Le façonnage de la critique par les dispositifs. Le cas du débat sur les nanotechnologies ». Semen 39, 39-58

Duman, Duygu Çurum. 2012. «L'identité et ses représentations : éthos et pathos, Synergies Turquie $5,187-200$

Frank, David A. 2003. « The Jewish Countermodel: Talmudic argumentation, the New Rhetoric Project, and the Classical Tradition of Rhetoric », JRC 26, 163-194

Gauthier, Gilles. 1995. «L'argumentation périphérique dans la communication politique. Le cas de l'argument ad hominem », Hermès 16, 167-185

Krieg-Planque, Alice. 2012. Analyser les discours institutionnels (Paris : Colin) 
Krieg-Planque Alice \& Claire Oger. 2017. «Éléments de langage ». Publictionnaire. Dictionnaire encyclopédique et critique des publics. <http://publictionnaire.huma-num.fr/notice/elements-delangage/>

Kriszan, Attila. 2011. "The EU is not them, but us". The First Person Plural and the Articulation of Collective Identities in European Political Discourse (Cambridge : Cambridge Scholar Publishing)

Landowski, Éric. 1997. Présence de l'autre. Essais de socio-sémiotique II (Paris : PUF)

Le Bart, Christian. 2014. « Les conditions de production du discours politique ». Recherches en communication 41, 35-46

Maingueneau, Dominique. 2007. « Genres de discours et modes de généricité ». Le français aujourd'hui, 159, 29-35

Mortureux, Marie-Françoise. 2004. La lexicologie entre langue et discours (Paris : Colin)

Navarro, Julien. 2009. «Les rôles au Parlement européen. Une typologie des pratiques de représentation », Revue française de Science politique 2009-3, 479-506

Née, Émilie, Frédérique Sitri \& Marie Véniard. 2016. «Les routines, une catégorie pour l'analyse de discours : le cas des rapports éducatifs ", Lidil 53, 71-92

Oger, Claire. 2012. « La conflictualité en discours : le recours à l'injure dans les arènes publiques », Argumentation et Analyse du Discours 8, 1-15

Oger, Claire \& Caroline Ollivier-Yaniv. 2003. « Du discours de l'institution aux discours institutionnels : vers la constitution de corpus hétérogènes ", communication au $X^{e}$ Colloque bilatéral franco-roumain, CIFSIC Université de Bucarest, 28 juin-3 juillet $2003<\mathrm{http}$ :// archivesic.ccsd.cnrs.fr/sic_00000717>

Oger, Claire \& Caroline Ollivier-Yaniv. 2006. «Conjurer le désordre discursif. Les procédés de "lissage" dans la fabrication du discours institutionnel », Mots 81, 63-77

Ollivier-Yaniv, Caroline. 2011. «Les 'petites phrases' et 'éléments de langage' : des catégories en tension ou l'impossible contrôle de la parole par les spécialistes de la communication ", Communication \& Langages 168, 57-68

Orkibi, Eithan. 2015. «Le(s) discours de l'action collective : contextes, dynamiques et traditions de recherche ", Argumentation et Analyse du Discours 14, http://aad.revues.org/2002

Rabatel. Alain. 2015. «Une analyse du manifeste 'Pour des universités à la hauteur de leurs missions'. Pour une alternative à la gestion libérale des universités et de la recherche en Europe ». Semen 39, 59-80

Raus, Rachele. 2009. «L'éthos entre discours théoriques et typologies empiriques en politique », Studi e Ricerche 4, 251-263

Raus, Rachele. 2015. « Types de contre-discours et remaniements 'codiscursifs' : l'inscription du dit d'ATTAC et du LEF dans les rapports du Parlement européen sur les femmes (2004-2012), Semen $39,115-134$

Raus, Rachele. 2017. « Les rapports d'initiative au Parlement européen ou comment la traduction influe sur les aspects performatifs d'un genre discursif ». Mots 114, 95-115

Rizzitelli, Clara. 2012. La donna migrante nell'UE in una prospettiva italo-francese, Quanderni di Donna \& Ricerca 26, <https://www.cirsde.unito.it/sites/c555/files/allegatiparagrafo/23-05-2016/ quaderno_rizzitelli.pdf> 
Viktorovitch, Clément. 2010. «Les commissions parlementaires à l'assemblée nationale et au sénat : un havre de paix ? ", Parlement [s], Revue d'histoire politique, 14, 90-110

\section{Sources premières}

Fotyga, Anna Elżbieta. 2016. Rapport sur la communication stratégique de l'Union visant à contrer la propagande dirigée contre elle par des tiers. PE582.060v03-00

Gahler, Michael. 2017. Rapport sur le rapport annuel sur la mise en œuvre de la politique de sécurité et de défense commune. PE609.294v02-00

Honeyball, Mary. 2014. Rapport sur l'exploitation sexuelle et la prostitution et leurs conséquences sur l'égalité entre les hommes et les femmes. PE519.748v03-00

Honeyball, Mary. 2016. Rapport sur la situation des réfugiées et demandeuses d'asile dans l'Union européenne. PE571.702v03-00

Le Grop, Constance. 2017. Rapport sur la nécessité d'une stratégie de l'Union européenne pour éradiquer et prévenir l'écart entre les pensions des hommes et des femmes. PE589.332v03-00

McAllister, David. 2017. Rapport annuel sur la mise en cuvre de la politique étrangère et de sécurité commune. PE607.921v02-00

Noichl, Maria. 2015. Rapport sur la stratégie de l'Union européenne pour l'égalité entre les femmes et les hommes après 2015. PE 549.092v02-00

Paet, Urmas. 2016. Rapport sur l'Union européenne de la défense. PE584.127v02-00

Revault d'Allonnes Bonnefoy, Christine \& Anna Maria Corazza Bildt. 2017. Rapport sur la proposition de décision du Conseil portant conclusion, par l'Union européenne, de la convention du Conseil de l'Europe sur la prévention et la lutte contre la violence à l'égard des femmes et de la violence domestique. PE601.176v02-00

Verhofstadt, Guy. 2016. Rapport sur les évolutions et adaptations possibles de la structure institutionnelle actuelle de l'Union européenne. PE585.741v02-00

Záborská, Anna. 2015. Rapport sur l'application de la directive 2006/54/CE du Parlement européen et du Conseil du 5 juillet 2006 relative à la mise en œuvre du principe de l'égalité des chances et de l'égalité de traitement entre hommes et femmes en matière d'emploi et de travail. PE549.388v02-00

Ždanoka Tatjana \& Vilija Blinkevičiūtè. 2016. Rapport sur la mise en place de conditions sur le marché du travail favorisant l'équilibre entre la vie professionnelle et la vie privée. PE580.714v02-00

\section{NOTES}

1. Pour les familles d'arguments nous renvoyons à Philippe Breton (2015: 50).

2. D'après les auteurs, " $[\mathrm{u}]$ ne routine discursive consiste en la mise en relation de séquences linguistiques récurrentes, partiellement figées (i.e. les patrons définis plus haut) avec des déterminations discursives et des fonctions textuelles propres à un genre ou une sphère d'activité »

(Née, Sitri, Véniard $2016: 78$ ).

3. Il s'agit donc d'un contre-ethos « collectif». Tout comme l'éthos collectif, qui peut s'entendre comme « l'image du groupe » (Duman 2012 : 194), le contre-ethos collectif peut correspondre, en paraphrasant Charaudeau (2005: 90) « à une vision globale, mais [...] il n'est construit que par attribution apriorique, attribution d'une identité émanant d'une opinion collective vis-à-vis d'un groupe autre ». 
4. En ce sens, le cas de Záborská est d'autant plus intéressant que c'est justement elle l'auteure du rapport en question et que c'est donc son positionnement à devenir minoritaire à l'intérieur de sa propre commission.

5. Bien que l'étude de Julien Navarro concerne la cinquième législature du PE, et que donc les changements survenus entre temps puissent faire évoluer ces rôles, la figure du « contestataire » semble fort proche du «bouffon" (Landowski 1997), qui, dans la narration politique, met en cause les autres acteurs politiques et les institutions.

6. Rappelons que, selon Ruth Amossy (2014 : 176), la violence est un registre discursif qui peut s'ajouter à la polémique sans que pour cela elle lui soit constitutive. Soulignons également qu'en accord avec Claire Oger (2012), nous considérons la violence comme l'une des formes de l'argumentation polémique qui doit être posée sur un continuum par rapport à un modèle oratoire de l'argumentation, en dépassant donc toute opposition entre les deux.

7. Il s'agit du rapport Honeyball et donc de la commission des droits de la femme et de l'égalité des genres. Ce rapport porte sur les refugiées dans une perspective d'égalité, non de défense.

8. Citons, par exemple, les manifestes et les appels du Lobby européen des femmes (voir, entre autres, le manifeste sur « Les droits socio-économique des femmes et l'égalité femmes-hommes dans une perspective de cycle de vie " que les adhérents du LEF ont signé à Budapest le 11 mai 2012 ou l'appel de Bruxelles pour la libération de la prostitution du 4 décembre 2012) où justement, par la tournure «nous demandons ", ce lobby propose aux décideurs, notamment à l'Union européenne, des discours alternatifs.

9. C'est par exemple le cas du discours de la Commission de l'Union européenne ou d'acteurs externes (lobby, associations...) dont le discours est reconnu comme légitime par les institutions européennes, ce qui permet des relations codiscursives (Raus 2015) déclenchant la circulation et la reprise d'éléments langagiers et discursifs.

\section{RÉSUMÉS}

Dans le système actuel de la "démocratie de consensus ", la négociation du consensus semble s'appuyer sur une vision partagée, voire sur un ethos communautaire, qui permettrait de dépasser tout clivage idéologique. La présence des opinions minoritaires dans les rapports non législatifs du Parlement européen montre cependant que la polémique reste un élément fondamental du discours argumentatif institutionnel. Nous proposons d'examiner le rôle que joue l'avis minoritaire des eurodéputés dans le cadre de la négociation du consensus, à partir des cas de la commission des affaires étrangères et des droits de la femme et de l'égalité de genre au cours de la législature actuelle. La distinction entre deux types d'opinion, individuelle et collective, permet de montrer que, par-delà la présence constante de la contestation, le contrediscours élaboré par les groupes finit par produire un discours alternatif qui nourrit l'espace démocratique européen dans une opposition de valeurs et d'ethè.

In the current system of "consensus democracy", negotiated agreement seems to be achieved through a shared vision, a sort of "communitarian ethos" that can overcome any ideological rifts. The minority opinions contained in the European Parliament non-legislative reports show however that the institutional discourse is fundamentally based on controversy. This paper examines the role of minority opinions in the negotiation of a consensus through the analysis of some examples borrowed from the Committee of Foreign Affairs and from the Committee of 
Women's Rights and Gender Equality in the current Parliament. The differences between two kinds of opinions - individual or in-group - show that, in addition to a dissenting discourse, the in-group counter-discourse opposing values and ethè produces an alternative discourse that nourishes the European democratic space.

\section{INDEX}

Mots-clés : consensus, discours institutionnel, ethos communautaire, opinion minoritaire, Parlement européen

Keywords : communitarian ethos, consensus process, institutional discourse, European Parliament, minority opinion

\section{AUTEUR}

\section{RACHELE RAUS}

Università di Torino 